\title{
Clinical, Pathological, and Surgical Outcomes for Adult Pineoblastomas
}

Melissa Gener, $\mathrm{MD}^{1}$ : Andrew R. Conger, MD²: Jamie Van Gompel, MD; Mohammad S. Ariai, MD:

Mark Jentoft, $\mathrm{MD}^{5}$; Fredric B. Meyer, $\mathrm{MD}^{4}$; Jeremy S. Cardinal, $\mathrm{MD}^{3}$ : José M. Bonnin, MD¹; and Aaron

$$
\text { A. Cohen-Gadol, MD, } \mathrm{MSc}^{2,6}
$$

\section{Affiliations:}

1. Department of Pathology and Laboratory Medicine, Indiana University School of Medicine, Indianapolis, IN

2. Goodman Campbell Brain and Spine, Department of Neurological Surgery, Indiana University School of Medicine, Indianapolis, IN

3. Department of Radiology, Indiana University School of Medicine, Indianapolis, IN

4. Department of Neurosurgery, Mayo Clinic, Rochester, MN

5. Department of Pathology and Laboratory Medicine, Mayo Clinic. Rochester, MN

6. Indiana University Simon Cancer Center, Indianapolis, IN

\section{Email addresses:}

Melissa Gener: mgener@iupui.edu

Andrew R. Conger: aconge@lsuhsc.edu

Jamie Van Gompel: VanGompel.Jamie@mayo.edu

Mohammad S. Ariai: Ariai.MohammadShafie@mayo.edu

Mark Jentoft: Jentoft.Mark@mayo.edu

Fredric B. Meyer: Meyer.fredric@mayo.edu

Jeremy Cardinal: jscardin@iupui.edu

José Bonnin: jbonnin@iupui.edu

\section{Correspondence:}

Aaron A. Cohen-Gadol, MD, MSc

Goodman Campbell Brain and Spine,

Indiana University Department of Neurological Surgery

355 W 16th St. Ste 5100

Indianapolis, IN, 46202

Phone: 317-396-1300 Fax: 317-924-8472 acohenmd@gmail.com 


\section{Highlights}

Pineoblastomas, usually found in children, are exceedingly rare in adults. Few reports have compared aspects of these tumors between adults and children. This study found:

- $\quad$ Of the 12 patients in this series, only 5 died of their disease (average length of survival 118 months), and 5 patients are still alive with no evidence of disease (average length of follow-up, 92 months).

- Patients with subtotal resections or diagnostic biopsies did not suffer a worse prognosis.

- Progression-free survival and overall survival are much higher for adult patients than for children

- Data suggest that pineoblastomas have a less aggressive clinical course in adults than in children.

Funding and conflicts of interest: No funding was received for this study and the authors have no conflicts of interest.

Short title: Pineoblastomas

Key Words: neoplasms; outcome; pathology; pineoblastoma; resection

\section{Abstract}

Introduction: Pineoblastomas are uncommon primitive neurectodermal tumors and most occur in children. They are exceedingly rare in adults. Few published reports have compared the various aspects of these tumors between adults and children.

Methods: The authors report a series of 12 pineoblastomas in adults from 2 institutions over 24 years. The clinical, radiologic, and pathologic features, and clinical outcomes were compared with previously reported cases in children and adults.

Results: Patients ranged from 24-81 years of age, and all but 1 presented with symptoms of obstructive hydrocephalus. Three patients underwent gross total resection, while subtotal resection was performed in 3. Diagnostic biopsies were obtained in another 6 patients. Pathologically, the tumors had the classical morphological and immunohistochemical features of pineoblastomas. Postoperatively, 10 patients received radiotherapy and 5 received chemotherapy. Compared with previously reported cases, several differences were noted in clinical outcomes. Of the 12 patients, only 5 (42\%) died of their disease (average length of survival, 118 months), another 5 (42\%) patients are alive with no evidence of disease (average length of follow-up, 92 months). One patient died of unrelated causes and 1 was lost to followup. Patients with subtotal resections or diagnostic biopsies did not suffer a worse prognosis. Of the 9 
patients with biopsy or subtotal resection, 4 are alive, 4 died of their disease, and 1 died of an unrelated hemorrhagic cerebral infarction.

Conclusions: Although this series is small, the data suggest that pineoblastomas in adults have a less aggressive clinical course than in children. 


\section{Introduction}

Primary pineal parenchymal tumors are rare and account for $0.4 \%$ to $1 \%$ of primary central nervous system neoplasms in all age groups combined (7). Approximately 25-50\% of primary pineal parenchymal tumors are pineoblastomas $(20,29)$, WHO grade IV. These are embryonal tumors that arise in the region of the pineal gland, are found preferentially in children, and often disseminate extensively along the cerebrospinal fluid (CSF) pathways. Patients with pineoblastomas have a reported 5-year survival rate of only $10 \%(1,11)$. Only a few isolated case reports and small series of these tumors occurring in adults have been documented in the literature $(4,7,17,21)$. In this report, we discuss the largest series to date of 12 cases of pineoblastomas occurring in patients older than 19 years of age who presented at our institutions during the last 24 years.

We aim to determine if there are specific pathologic, clinical, radiologic features, or surgical techniques that are associated with outcome.

\section{Materials and Methods}

We extracted information from the electronic medical records at Indiana University Health and Mayo Clinic, using the key words "pineal gland,” "pineoblastomas,” "pineal parenchymal tumor,” and “pineal gland tumor.” Data about the cases were transferred into a Microsoft Excel 2008 spreadsheet.

The following information was obtained about each patient: sex, age, race, institution, tissue source, specimen type, presenting symptoms and their duration, presence of hydrocephalus, tumor location and size, tumor invasion, spread of tumor, history of previous resection, surgical approach for resection, extent of resection (percentage), postoperative complications, the need for a shunt, adjuvant radiation, other adjuvant therapy, follow-up time, and cause of death. Patients younger than 19 years of age were defined as children and excluded from the study. The clinical presentation, radiological features, histopathological findings, treatments, and survival for all adult patients with primitive neuroectodermal tumors, WHO grade IV, in the pineal region were reviewed in detail.

A staff pathologist examined all of the pathology case materials to determine the final diagnosis. Tumors were examined for histologic characteristics, mitotic rate, Ki67 (MIB-1) proliferative index, synaptophysin, neurofilament protein and glial acidic fibrillary protein immunoreactivity, and the presence of rosettes. We also reviewed the available radiologic studies for each patient to determine if there were specific imaging characteristics in these tumors in adults that differ from those in children. Sum of squares for error was employed to determine the Kaplan Myers survival curves comparing the progression-free and overall survival of the patients using a variety of parameters. 


\section{Results}

Our data search spanned the last 24 years at our 2 institutions. Twelve adult patients were diagnosed with pinealoblastomas during 1990-2013. These patients included 2 men and 10 women (range 24-81 years of age at diagnosis). The median age at diagnosis was 47 years. The average clinical followup time was 95 months (range 0-288 months).

The most common clinical presentation was headaches. Additional presenting symptoms included blurred vision, vertigo, urinary incontinence, back pain, weight loss, gait disturbances, and lower extremity weakness (Table 1). Hydrocephalus was present as an initial finding in all but one of our patients.

\section{Radiographic Features}

The reports of imaging studies were available for all 12 patients in our series. Eleven of the 12 patients presented with obstructive hydrocephalus. The imaging reports were available on all patients and 3 patients had available imaging studies that were also reviewed by our radiologists (Figures 1 and 2). Eleven patients demonstrated pineal masses at presentation. One patient presented with obstructive hydrocephalus, but no appreciable mass found on noncontrast computed tomography (CT) imaging. Three months after initial presentation, follow-up imaging on this patient demonstrated a 2-cm pineal mass.

The reviews of the available imaging studies in 3 patients demonstrated that the smallest mass was ovoid, whereas the other masses were slightly lobulated with mildly irregular margins. Two were well defined on postcontrast MR images, and one appeared mildly infiltrative. One mass was T1-isointense and T2-isointense; one was T1-isointense and T2-hyperintense; and a third was mildly T1-hypointense and T2-hyperintense compared with gray matter. All 3 masses demonstrated avid heterogeneous patterns of enhancement. Two masses demonstrated mild diffusion restriction diffusely. Diffusion-weighted imaging was not performed on the third case. One mass had no cystic components; one demonstrated a few very small internal cysts; and the third demonstrated a moderate size central cystic cavity. Two of the 3 patients had preoperative CT performed, and neither of them demonstrated the classically described “exploded” pineal calcifications. Rather, both demonstrated a single cluster of displaced pineal calcifications.

The imaging findings in our series conform to the previously described typical imaging characteristics, with the exceptions of the tumor that was too small to detect at presentation and the displaced (rather than exploded) pineal calcifications in 2 cases. Diffusion restriction was demonstrated in both cases that had diffusion-weighted images available. Diffusion restriction 
has been suggested as a possible distinguishing characteristic at diagnosis (9) and a possible tumor marker at posttreatment follow-up (13).

\section{Treatment and Survival}

Initial surgical treatment data were available for all 12 of our patients. Six patients underwent stereotactic biopsies only. Two patients had a subtotal resection $(<90 \%$ of tumor removed); 1 patient had a near total gross resection ( $>90 \%$ of tumor removed); and 3 patients underwent gross total resections. For patients undergoing resections, the tumors were exposed via suboccipital supracerebellar approach. Table 2 summarizes the surgical technique and extent of resection for each patient.

Among the 12 patients in our series, 10 received postsurgical radiation therapy. In addition, 5 patients completed chemotherapy. These results are shown in Table 3.

Follow-up information was available for 11 of our patients. The median patient followup time was 95 months. Three of our patients suffered tumor recurrences. Two had local recurrence, and 1 had a T10 spinal metastasis with no evidence of residual pineal disease. The median time from diagnosis to recurrence was 144 months (12 years). All 3 patients with recurrences died of their disease. The median time from recurrence to death from disease was 48 months. In addition, 2 patients died directly from their disease shortly after diagnosis because of increased intracranial pressure caused by tumor mass effect, whereas another died of infection and intraparenchymal hemorrhage not related to the tumor mass itself (unrelated stroke). The time from initial diagnosis to death for these 3 patients ranged for 0.5 to 11 months. The remaining 5 patients had an average follow-up time of 92 months and all have had no incidence of recurrence.

\section{Pathologic Features}

Pathology reports were available for all 12 patients, and original histological sections were available for review in 6 (Table 4). Histologic characteristics of the tumors in all 12 patients were consistent with the previously described morphologic and immunohistochemical phenotype in pinealoblastomas occurring in both children and adults.

All of the examined tissues showed the classically described features of a pineoblastoma. They are highly cellular tumors composed of monotonous overlapping hyperchromatic, round to oval cells with salt and pepper chromatin, and with inconspicuous to no nucleoli and scant eosinophilic cytoplasm. Occasional rosettes were identified (Figure 3). Notably, all of our 
patients showed strong and diffuse immunoreactivity for synaptophysin and no immunoreactivity for glial acidic fibrillary protein (Figure 4). The tumors had varied mitotic rates and Ki67 proliferative indices. The lowest reported mitosis rate was 3/10 high power field (HPF) and the highest was 32/10 HPF. We examined the mitotic rate and proliferative indices to determine if tumors with a higher proliferation or mitotic rate corresponded with a more aggressive clinical course.

In our evaluation of the histologic characteristics of these tumors, we found that the reliable predictors of a worse clinical outcome is an increase in mitotic count (>10/10 HPF) and an elevated (>10\%) Ki67 proliferative index. Patients with either of these two findings had a worse progression-free survival and overall survival. Patients were divided into 2 groups for each of the 2 criteria. For mitotic rate, those with greater than 10 mitoses per 10 HFP were compared with those with less than or equal to 10 mitoses per 10 HPF. The results of the Kaplan Myers survival curves are seen in Figures 5 and 6. For Ki67 proliferative index, those with a rate greater than $10 \%$ were compared to those with a rate of less than or equal to $10 \%$. Those results are seen in Figures 7 and 8.

Patient age at diagnosis was also examined to determine if a certain age group had worse overall survival. We divided our patients into age categories, 19- 29, 30-39. 40-49, 50-59, and 60 years of age and older. The results for patient survival are shown in Table 5.

\section{Discussion}

There is some available literature addressing the clinical outcomes of pineoblastomas in adult patients. Since 1979, approximately 200 cases have been reported, some of these relevant series include groups of patients with heterogeneous pathologies such as different pineal region tumors, therefore, the exact number of adult patients with pathologically confirmed pineoblastoma is not completely certain. The largest study to date is from the United Kingdom and included 95 patients age 16 and older (30). Before that study, 2 other studies from Japan (17) and Germany (21) included 34 and 64 patients, respectively. A more recent study from MD Anderson Cancer Center (10) reported 14 adult patients. The important findings and patient demographics from each of these studies are presented in Table 6.

Lutterbach and colleagues presented 101 patients, age 18 and older, from multiple centers, many of whom had been previously reported (2,3,5-8,12,15,16,18,19,22-26,28,29,3235). These cases included both pineoblastomas and pineal parenchymal tumors of intermediate 
differentiation. Sixty-four patients harbored pineoblastomas, including both those in the review of all published literature from 1979 to 2002 and newly reported cases. The median survival of these patients was 77 months. Factors that increased survival included completeness of resection and extent of disease. As in our study, age and sex did not affect survival outcomes.

Lee and colleagues presented 34 patients, age 16 and older, from the Brain Tumor Registry of Japan. In this study, the number of patients in the 16-18 age group is not identifiable, making it difficult to draw a direct comparison of their results to ours. The median survival was 25.7 months. Upon analysis, 2 variables were noted to independently affect survival: completeness of resection and dose of cranial radiation. No patients with gross total resection died, and those receiving greater or equal to $40 \mathrm{~Gy}$ of radiation benefitted from a statistically significant survival advantage, regardless of extent of resection.

Selvanathan et al (30) published the largest series to date in 2012 using the Surveillance, Epidemiology, and End Results (SEER) database from the United Kingdom. Included were 95 patients age 16 years and older. Again, as with the study by Lee and colleagues (17), the authors did not divulge the number of their patients in the 16 -18 age group, making direct comparison of their results to ours difficult. They concluded that they only independent predictors of a better outcome were age at diagnosis and the presence of localized disease. Interestingly, as in our series, they did not see worse survival rates in patients who received subtotal resections or biopsies only.

Based on our 12 cases, we reviewed our patients’ age, sex, clinical presentation, histologic findings, radiographic features, surgical approach, and radiation/chemotherapy treatment to determine if there are specific aspects of pineoblastomas in adults that are different from those in children.

In children, pineal parenchymal tumors have an equal male and female distribution. However, our adult patients with pinealoblastomas included 10 women and 2 men. In our review of the literature, 4 series showed a predominance of males $(7,10,17,21)$ and 2 studies showed a slight predominance of females $(11,30)$. Because of the rarity of these tumors, it is unlikely that we can predict if these tumors in adults are reliably associated with gender predominance.

The only histological characteristic that was statistically significant in predicting a worse progression-free survival was the mitotic count of $>10$ mitotic figures per 10 high-power fields (HPF). Although the difference in the Ki67 proliferate index between those with a rate of $\leq 10 \%$ 
versus those with a rate $>10 \%$ was not statistically significant, the data demonstrate some difference in these 2 groups overall and in progression-free survivals. The small sample size may account for the lack of statistical significance for these differences. Overall, mitotic rate and Ki67 proliferative indices correlate well in terms of predicting the growth potential of these tumors.

The imaging characteristics of pineoblastomas have been previously described $(9,23,27,31)$. Pineoblastomas are often large $(>3 \mathrm{~cm})$ at presentation, and cause obstructive hydrocephalus in nearly all patients. Computed tomography images typically demonstrate a hyperattenuating mass, which reflects tumor hypercellularity. Pineal calcifications, if present, are often "exploded” and displaced to the periphery of the mass. MR imaging typically reveals a heterogeneous mass with T1 signal hypointense to isointense, and T2 signal isointense to hyperintense relative to gray matter, and often heterogeneous enhancement following administration of contrast media. Diffusion restriction may be seen, likely related to hypercellularity. Necrosis and hemorrhage may be present, but extensive cystic change is rare. Cerebrospinal fluid dissemination is common, and the entire craniospinal axis should be imaged when pineoblastoma is considered in the differential diagnosis.

Considerable overlap exists between the imaging appearance of pineoblastoma and lower grade primary pineal neoplasms $(23,27,31)$. Germinomas, commonly in the differential diagnosis of pineal tumors, also have overlap in imaging appearance, but have been found to demonstrate less diffusion restriction (9).

All but one of our patients presented with radiographic and clinical signs of hydrocephalus, including headache, double vision, blurry vision, and obtundation. This is the most common presenting feature in all cases of pineal parenchymal tumors, among both adults and children. Because all but one of our patients had hydrocephalus on presentation, we did not evaluate hydrocephalus as an independent prognostic feature. In the other small case series $(4,7,17,21,30)$, hydrocephalus was also the most common presenting feature, with the percentage of symptomatic patients ranging from 46-100\%.

One of the most striking clinical differences that we observed between our patients and the reported pediatric patients is that progression-free survival and overall survival for adult patients are much higher than for children. Our patients, on average, had a 109-month progression-free survival among the 8 patients with clinical follow-up who did not die of their 
initial disease. In addition, after recurrence, our patients still lived, on average, 36 months from the time of recurrence. In the literature, death in children with this disease is usually very rapid after relapse (14).

Also in contrast with pediatric patients, in whom gross total resection of the main tumor is linked to significantly better progression-free and overall survival, we observed that 3 of our 6 patients who underwent diagnostic biopsy without formal resection were alive and free of their disease at their last follow-up visit. These patients received a combination of radiotherapy and chemotherapy after diagnosis. However, our patient with spinal metastasis eventually died of the disease. His initial diagnosis was also made only by biopsy, and his spinal metastasis was not seen until 17 years after the initial diagnosis.

\section{Conclusions}

A review of our 12 patients with this rare disease suggests that pineoblastomas in adults have a less aggressive clinical course than those in the pediatric population. Safe surgical resection and adjuvant therapy are associated with reasonable outcomes. 


\section{References}

1. Al-Hussaini M, Sultan I, Abuirmileh N, Jaradat I, Qaddoumi I: Pineal gland tumors: experience from the SEER database. J Neurooncol 94:351-358, 2009

2. Ashley DM, Longee D, Tien R, Fuchs H, Graham ML, Kurtzberg J, Casey J, Olson J, Meier L, Ferrell L, Kerby T, Duncan-Brown M, Stewart E, Colvin OM, Pipas JM, McCowage G, McLendon R, Bigner DD, Friedman HS: Treatment of patients with pineoblastoma with high dose cyclophosphamide. Med Pediatr Oncol 26:387-392, 1996

3. Barlas O, Bayindir C, Imer M, Ayan I, Darendeliler E: Non-resective management of pineoblastoma. Minim Invasive Neurosurg 43:163-170, 2000

4. Behdad A, Perry A: Central nervous system primitive neuroectodermal tumors: a clinicopathologic and genetic study of 33 cases. Brain Pathol 20:441-450, 2010

5. Borit A, Blackwood W, Mair WG: The separation of pineocytoma from pineoblastoma. Cancer 45:1408-1418, 1980

6. Brockmeyer DL, Walker ML, Thompson G, Fults DW: Astrocytoma and pineoblastoma arising sequentially in the fourth ventricle of the same patient. Case report and molecular analysis. Pediatr Neurosurg 26:36-40, 1997

7. Chang SM, Lillis-Hearne PK, Larson DA, Wara WM, Bollen AW, Prados MD: Pineoblastoma in adults. Neurosurgery 37:383-390; discussion 390-381, 1995

8. Charafe-Jauffret E, Lehmann G, Fauchon F, Michiels JF, Paquis P, Maraninchi D, Hassoun J: Vertebral metastases from pineoblastoma. Arch Pathol Lab Med 125:939943, 2001

9. Dumrongpisutikul N, Intrapiromkul J, Yousem DM: Distinguishing between germinomas and pineal cell tumors on MR imaging. AJNR Am J Neuroradiol 33:550-555, 2012

10. Farnia B, Allen PK, Brown PD, Khatua S, Levine NB, Li J, Penas-Prado M, Mahajan A, Ghia AJ: Clinical outcomes and patterns of failure in pineoblastoma: A 30-year, singleinstitution retrospective review. World Neurosurg 82:1232-1241, 2014

11. Fauchon F, Jouvet A, Paquis P, Saint-Pierre G, Mottolese C, Ben Hassel M, Chauveinc L, Sichez JP, Philippon J, Schlienger M, Bouffet E: Parenchymal pineal tumors: a clinicopathological study of 76 cases. Int J Radiat Oncol Biol Phys 46:959-968, 2000 
12. Fuller BG, Kapp DS, Cox R: Radiation therapy of pineal region tumors: 25 new cases and a review of 208 previously reported cases. Int J Radiat Oncol Biol Phys 28:229245, 1994

13. Gasparetto EL, Cruz Jr LC, Doring TM, Araujo B, Dantas MA, Chimelli L, Domingues RC: Diffusion-weighted MR images and pineoblastoma: diagnosis and follow-up. Arq Neuropsiquiatr 66:64-68, 2008

14. Gilheeney SW, Saad A, Chi S, Turner C, Ullrich NJ, Goumnerova L, Scott RM, Marcus K, Lehman L, De Girolami U, Kieran MW: Outcome of pediatric pineoblastoma after surgery, radiation and chemotherapy. J Neurooncol 89:89-95, 2008

15. Jacobs JJ, Rosenberg AE: Extracranial skeletal metastasis from a pinealoblastoma. A case report and review of the literature. Clin Orthop Relat Res:256-260, 1989

16. Jooma R, Kendall BE: Diagnosis and management of pineal tumors. J Neurosurg 58:654-665, 1983

17. Lee JY, Wakabayashi T, Yoshida J: Management and survival of pineoblastoma: an analysis of 34 adults from the Brain Tumor Registry of Japan. Neurol Med Chir (Tokyo) 45:132-141; discussion 141-132, 2005

18. Lesnick JE, Chayt KJ, Bruce DA, Rorke LB, Trojanowski J, Savino PJ, Schatz NJ: Familial pineoblastoma. Report of two cases. J Neurosurg 62:930-932, 1985

19. Linggood RM, Chapman PH: Pineal tumors. J Neurooncol 12:85-91, 1992

20. Louis DN, Ohgaki H, Wiestler OD, Cavenee WK, Burger PC, Jouvet A, Scheithauer BW, Kleihues P: The 2007 WHO classification of tumours of the central nervous system. Acta Neuropathologica 114:97-109, 2007

21. Lutterbach J, Fauchon F, Schild SE, Chang SM, Pagenstecher A, Volk B, Ostertag C, Momm F, Jouvet A: Malignant pineal parenchymal tumors in adult patients: patterns of care and prognostic factors. Neurosurgery 51:44-55; discussion 55-46, 2002

22. Matsumoto K, Higashi H, Tomita S, Ohmoto T: Pineal region tumours treated with interstitial brachytherapy with low activity sources (192-iridium). Acta Neurochir (Wien) 136:21-28, 1995

23. Nakamura M, Saeki N, Iwadate Y, Sunami K, Osato K, Yamaura A: Neuroradiological characteristics of pineocytoma and pineoblastoma. Neuroradiology 42:509-514, 2000 
24. Neuwelt EA, Glasberg M, Frenkel E, Clark WK: Malignant pineal region tumors. A clinico-pathological study. J Neurosurg 51:597-607, 1979

25. Patil AA, Good R, Bashir R, Etemadrezaie H: Nonresective treatment of pineoblastoma: a case report. Surg Neurol 44:386-903; discussion 390-381, 1995

26. Paulino AC, Melian E: Medulloblastoma and supratentorial primitive neuroectodermal tumors: an institutional experience. Cancer 86:142-148, 1999

27. Reis F, Faria AV, Zanardi VA, Menezes JR, Cendes F, Queiroz LS: Neuroimaging in pineal tumors. J Neuroimaging 16:52-58, 2006

28. Schild SE, Scheithauer BW, Haddock MG, Wong WW, Lyons MK, Marks LB, Norman MG, Burger PC: Histologically confirmed pineal tumors and other germ cell tumors of the brain. Cancer 78:2564-2571, 1996

29. Schild SE, Scheithauer BW, Schomberg PJ, Hook CC, Kelly PJ, Frick L, Robinow JS, Buskirk SJ: Pineal parenchymal tumors. Clinical, pathologic, and therapeutic aspects. Cancer 72:870-880, 1993

30. Selvanathan SK, Hammouche S, Smethurst W, Salminen HJ, Jenkinson MD: Outcome and prognostic features in adult pineoblastomas: analysis of cases from the SEER database. Acta Neurochir (Wien) 154:863-869, 2012

31. Smith AB, Rushing EJ, Smirniotopoulos JG: From the archives of the AFIP: lesions of the pineal region: radiologic-pathologic correlation. Radiographics 30:2001-2020, 2010

32. Tada E, Matsumoto K, Tomita S, Furuta T, Ohmoto T: [Usefulness of neoadjuvant brachytherapy in the treatment of pineoblastoma: a case report]. No Shinkei Geka 24:481-485, 1996

33. Tsumanuma I, Tanaka R, Washiyama K: Clinicopathological study of pineal parenchymal tumors: correlation between histopathological features, proliferative potential, and prognosis. Brain Tumor Pathol 16:61-68, 1999

34. Uematsu Y, Itakura T, Hayashi S, Komai N: Pineoblastoma with an unusually long survival. Case report. J Neurosurg 69:287-291, 1988

35. Vaquero J, Ramiro J, Martinez R, Bravo G: Neurosurgical experience with tumours of the pineal region at Clinica Puerta de Hierro. Acta Neurochir (Wien) 116:23-32, 1992 


\section{Figure Legends}

Figure 1: Pineoblastoma in a 51-year-old woman demonstrating mild diffusion restriction. Diffusion-weighted image demonstrates hyperintensity diffusely within the tumor relative to the cortex (left image). The apparent diffusion coefficient (ADC) map demonstrates isointensity within the tumor (middle image). The T2-weighted image demonstrates mild hyperintensity within the tumor (right image).

Figure 2: Pineoblastoma with a central cystic component in a 40-year-old woman. T1-weighted image demonstrates isointensity relative to gray matter within the solid portions of the tumor (left image). Heterogeneous enhancement is demonstrated within the solid portions of the lesion on the post-contrast T1-weighted image (middle image). A central cystic component is demonstrated on the T2-weighted image (right image).

Figure 3: Hypercellular tumor with uniform cells with high nuclear-to-cytoplasmic ratio (left upper image). Mitoses are abundant (right upper and left lower images). Homer-Wright rosettes are rare, but usually seen in pineoblastomas (right lower image).

Figure 4: Glial acidic fibrillary protein immunostain demonstrated reactivity in the surrounding and infiltrated glial tissue. Immunoreactivity is absent in the tumor cells (left image). Synaptophysin is strongly immunoreactive in all tumor cells (right image).

Figure 5. Progression-free survival with reference to mitotic rate (MR) (9). Patients with MR $\leq$ 10/10 HPF had a median progression-free survival of 84 months. Patients with MR >10/10 HPF showed a median progression-free survival of 3 months.

Stats: Log-Rank $X^{2}=5.0000, \mathrm{p}=0.025$

Figure 6. Overall survival with reference to mitotic rate (MR). Patients with $M R \leq 10 \%$ had a median overall survival of 108 months (average $=139.0$ months). Patients with MR $>10 \%$ had a median overall survival of 3 months (average $=3.5$ months). $\log$-Rank $X^{2}=0.8744, p=0.350$ Figure 7. Progression-free survival with reference to Ki67 proliferative index. Patients with Ki67 $\leq 10 \%$ had a median progression-free survival of 144 months (average $=128.2$ ). Patients with Ki67 $>10 \%$ had a median progression-free survival of 4 months (average $=30.3$ ). LogRank $X^{2}=1.1825, \mathrm{p}=0.277$

Figure 8. Overall survival with reference to Ki67 proliferative index. Patients with Ki67 $\leq 10 \%$ had a median overall survival of 180 months (average $=140.2$ months). Patients with Ki67 > $10 \%$ had a median overall survival of 4 months (average $=55.8$ months). Log-Rank $X^{2}=0.7387$, $\mathrm{p}=0.39$. 